may have common solutions

$$
\left\|\begin{array}{cccccccccccc}
F_{r} & F_{s} & F_{t} & 0 & 0 & 0 & 0 & 0 & 0 & 0 & 0 & 0 \\
0 & F_{r} & F_{s} & F_{t} & 0 & 0 & 0 & 0 & 0 & 0 & 0 & 0 \\
0 & 0 & 0 & 0 & F_{r} & F_{s}^{\prime} & F_{t} & 0 & 0 & 0 & 0 & 0 \\
0 & 0 & 0 & 0 & 0 & F_{r} & F_{s} & F_{t} & 0 & 0 & 0 & 0 \\
0 & 0 & 0 & 0 & 0 & 0 & 0 & 0 & F_{r} & F_{s} & F_{t} & 0 \\
0 & 0 & 0 & 0 & 0 & 0 & 0 & 0 & 0 & F_{r} & F_{s} & F_{t} \\
G_{r} & G_{s} & G_{t} & 0 & H_{r} & H_{s} & H_{t} & 0 & K_{r} & K_{s} & K_{t} & 0 \\
0 & G_{r} & G_{s} & G_{t} & 0 & H_{r} & H_{s} & H_{t} & 0 & K_{r} & K_{s} & K_{t}
\end{array}\right\|=0 .
$$

And since

$$
(F, G)=(F, H)=(F, K)=(G, H)=(G, K)=(H K)=0
$$

for the same value of $\mu: \nu$, we have, in addition to the vanishing of the above matrix,

$$
\begin{aligned}
& \frac{F_{r}\left(G_{x}\right)-G_{r}\left(F_{x}\right)}{F_{t}\left(G_{y}\right)-G_{t}\left(F_{y}\right)}=\frac{F_{r}\left(H_{x}\right)-H_{r}\left(F_{x}\right)}{F_{t}\left(H_{y}\right)-H_{t}\left(F_{y}^{\prime}\right)}=\frac{F_{r}\left(K_{x}\right)-K_{r}\left(F_{x}\right)}{F_{t}\left(K_{y}\right)-K_{t}\left(F_{y}^{\prime}\right)} \\
& =\frac{G_{r}\left(H_{x}\right)-H_{r}\left(G_{x}\right)}{G_{t}\left(H_{y}\right)-H_{t}\left(G_{y}\right)}=\frac{G_{r}\left(K_{x}\right)-K_{r}\left(G_{x}\right)}{G_{t}\left(K_{y}\right)-K_{t}\left(G_{y}\right)}=\frac{H_{r}\left(K_{x}\right)-K_{r}\left(H_{x}\right)}{H_{t}\left(K_{y}\right)-K_{t}\left(H_{y}\right)} .
\end{aligned}
$$

Obviously the plan is general, and one could write down the necessary conditions that a system of $n$ partial differential equations of the type above considered should have solutions in common.

\title{
NOTE ON DETERMINANTS WHOSE TERMS ARE CERTAIN INTEGRALS.
}

BY PROFESSOR R. G. D. RICHARDSON AND MR. W. A. HURWITZ.

(Read before the American Mathematical Society, September 14, 1909.)

THE object of the present note is to prove two simple identities involving a determinant whose elements are certain integrals, and to mention some special cases. Determinants of the form considered present themselves in problems connected with linear differential and integral equations and the calculus of 
variations.* The theorems proved include in particular the well-known Schwarz's and Bessel's identities and inequalities, which are of value in the theory of linear integral equations. $\dagger$ A determinant involving infinite series in the same way in which the determinants here considered involve integrals is used by Schmidt in his theory of points and vectors of a function space. Expressions of the same character present themselves in the problem of finding, according to the criterion of the method of least squares, the nearest approximation to an arbitrary function in terms of given functions, not necessarily orthogonal.

1. Let $\phi_{1}, \phi_{2}, \ldots, \phi_{n} ; \psi_{1}, \psi_{2}, \ldots, \psi_{n}$ denote functions which are limited and integrable in a certain region $R$ of an m-dimensional space, or if unlimited, are such that the product of any function of the first set by any function of the second set is integrable; let $D_{i}$, $\bar{D}_{i}$ denote the determinant obtained by replacing respectively the ith row of the determinant

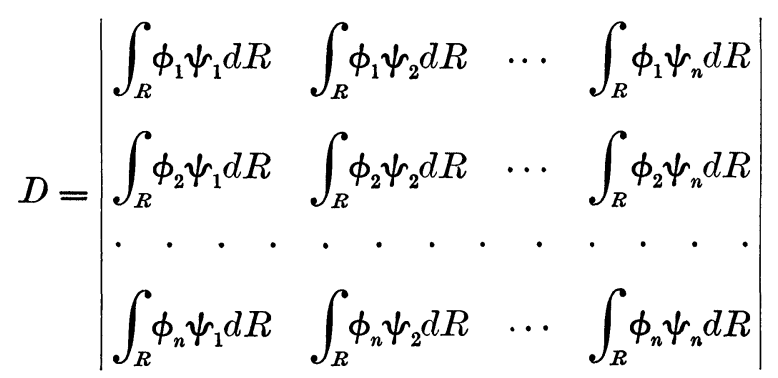

by $\psi_{1}, \psi_{2}, \ldots, \psi_{n}$ or the $j$ th column by $\phi_{1}, \phi_{2}, \ldots, \phi_{n}$, and let $\Delta_{i j}$ be the co-factor of that term of $D$ which stands in the ith row and the jth column. Then

$$
\int_{R} D_{i} \bar{D}_{j} d R=D \Delta_{i j}
$$

* For further identities which arise when the functions involved are known to be solutions of certain differential equations, see an article by Professor Richardson entitled "Das Jacobische Kriterium der Variationsrechnung und die Oscillationseigenschaften linearer Differentialgleichungen 2. Ordnung," shortly to appear in the Mathematische Annalen.

† Cf. Erhard Schmidt, " Zur Theorie der linearen und nichtlinearen Integralgleichungen," Mathematische Annalen, vol. 63 (1907), p. 433.

$\ddagger_{+}$"Über die Aufösung linearer Gleichungen mit unendlich vielen Unbekannten," Rendiconti del Circolo Matematico di Palermo, vol. 25 (1908), p. 62. 
The proof follows directly from the following applications of well-known theorems on the expansion of determinants :

For we have

$$
\begin{aligned}
& \sum_{q=1}^{n} \Delta_{r q} \int_{R} \phi_{p} \psi_{q} d R=\left\{\begin{array}{c}
0, p \neq r, \\
D, p=r,
\end{array}\right. \\
& \sum_{q=1}^{n} \Delta_{i q} \psi_{q}=D_{i}, \quad \sum_{p=1}^{n} \Delta_{p j} \phi_{p}=\bar{D}_{j} .
\end{aligned}
$$

$$
\begin{aligned}
\int_{R} D_{i} \bar{D}_{j} d R=\sum_{p=1}^{n} \sum_{q=1}^{n} \Delta_{i q} \Delta_{p j} \int_{R} \phi_{p} \psi_{q} d R \\
=\sum_{p=1}^{n} \Delta_{p j}\left[\sum \Delta_{i q} \int_{R} \phi_{p} \psi_{q} d R\right]=\Delta_{i j} D
\end{aligned}
$$

which was to be proved.

2. Let $R_{1}, R_{2}, \cdots, R_{n}$ denote $n$ equal regions in different $m$ dimensional spaces and $\phi_{i}\left(R_{j}\right), \psi_{i}\left(R_{j}\right)$ the functions $\phi_{i}, \psi_{i}$ defined in the region $R_{j}$. Then

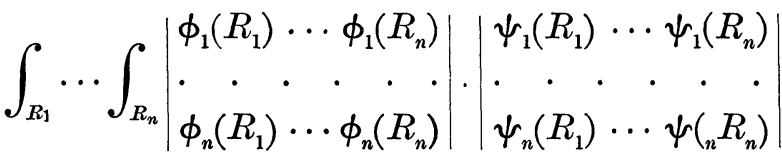

$$
\begin{aligned}
& \times d R_{1} \cdots d R_{n}=n ! D .
\end{aligned}
$$

In order to prove the theorem we note that the sign of any term $\phi_{i_{1}}\left(R_{k_{1}}\right) \cdot \phi_{i_{2}}\left(R_{k_{2}}\right) \cdots \phi_{i_{n}}\left(R_{k_{n}}\right)$ in the first determinant of the integrand, where each of the sequences $i_{1}, i_{2}, \ldots, i_{n}$; $k_{1}, k_{2}, \ldots, k_{n}$ is a permutation of the sequence of integers $1,2, \cdots, n$, is the power of -1 whose index is the number of transpositions required to build the substitution $\left(\begin{array}{lll}i_{1} i_{2} & \ldots & i_{n} \\ k_{1} k_{2} & \ldots & k_{n}\end{array}\right)-$ that is, to bring the term considered into the principal diagonal. Similarly the sign of the term $\psi_{j_{1}}\left(R_{k_{1}}\right) \cdot \psi_{j_{2}}\left(R_{k_{2}}\right) \cdots \psi_{j_{n}}\left(R_{k_{n}}\right)$ in the second determinant is the power of -1 whose index is the number of transpositions required to build the substitution $\left(\begin{array}{l}j_{1} j_{2} \ldots \ldots \\ k_{1} k_{2}\end{array} \ldots j_{n}\right)$. The result of integrating the product of these two terms is

$$
\begin{gathered}
\int_{R_{k_{1}}} \phi_{i_{1}}\left(R_{k_{1}}\right) \psi_{j_{1}}\left(R_{k_{1}}\right) d R_{k_{1}} \cdots \int_{R_{k_{n}}} \phi_{i_{n}}\left(R_{k_{n}}\right) \psi_{j_{n}}\left(R_{k_{n}}\right) d R_{k_{n}} \\
=\int_{R} \phi_{i_{1}} \psi_{j_{1}} d R \cdots \int_{R} \phi_{i_{n}} \psi_{j_{n}} d R
\end{gathered}
$$


to which is attached a sign equal to the power of -1 whose index is the sum of the numbers of transpositions required to build the substitutions $\left(\begin{array}{c}i_{1} i_{2} \ldots i_{n} \\ k_{1} k_{2} \ldots i_{n}\end{array}\right)$ and $\left(\begin{array}{c}j_{1} j_{2} \ldots j_{n} \\ k_{1} k_{2} \ldots k_{n}\end{array}\right)$; this sum furthermore is equal to the number of transpositions required to build the substitution $\left(\begin{array}{c}i_{1} i_{2} \ldots i_{n} \\ j_{1} j_{2} \ldots j_{n}\end{array}\right)$. The process of integration therefore leads to a term of $\mathrm{D}$ with the proper sign attached. By using every permutation of the sequence $(1,2, \cdots, n)$ for each of $\left(i_{1}, i_{2}, \cdots, i_{n}\right),\left(j_{1}, j_{2}, \cdots, j_{n}\right),\left(k_{1}, k_{2}, \cdots, k_{n}\right)$ we obtain the result of integrating every term in the product of the two determinants on the left of (2). By using every permutation of this sequence for each of $\left(i_{1}, i_{2}, \ldots, i_{n}\right),\left(j_{1}, j_{2}, \ldots, j_{n}\right)$, we obtain every term of $D$ once and only once for each choice of $\left(k_{1}, k_{2}\right.$, $\left.\ldots, k_{n}\right)$. We have thus the value of $D$ repeated as often as the number of the permutations of the sequence $(1,2, \cdots, n)$, - that is, $n$ ! times - which proves the theorem.

3. Both theorems reduce to interesting identities under various specializations. For example, if we take* $\psi_{1}=k \phi_{1}$, $\psi_{2}=k \phi_{2}, \cdots, \psi_{n}=k \phi_{n}$, we have from (1)

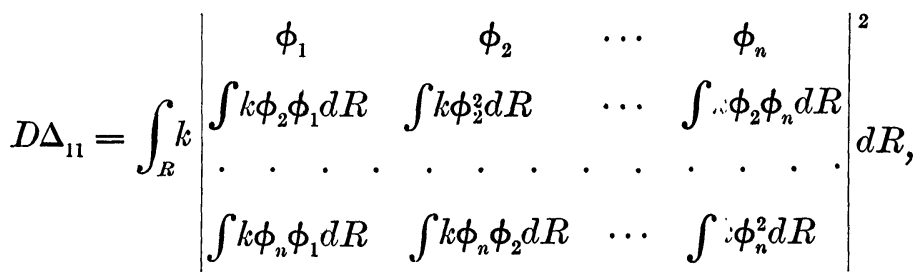

where

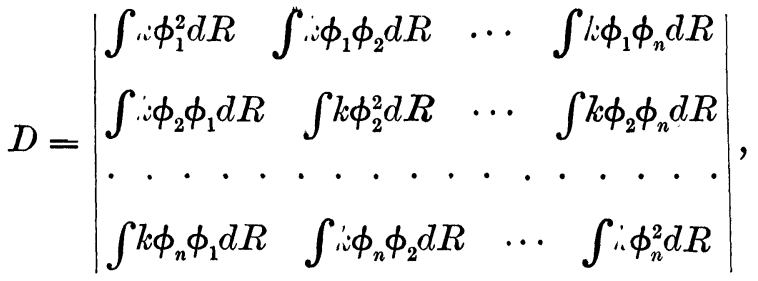

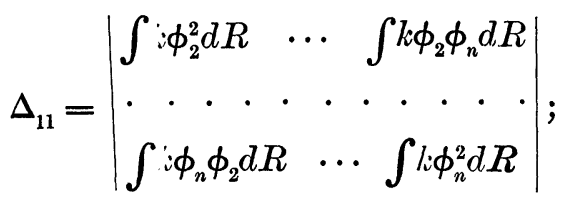

* In this case the restriction imposed above reduces to the condition that $k \phi_{i} \phi j$ is integrable $(i=1,2, \cdots, n ; j=1,2, \cdots, n)$. 
and from (2)

$$
\begin{aligned}
& D=\frac{1}{n !} \int_{R_{1}} \cdots \int_{R_{n}} k\left(R_{1}\right) \cdots k\left(R_{n}\right)
\end{aligned}
$$

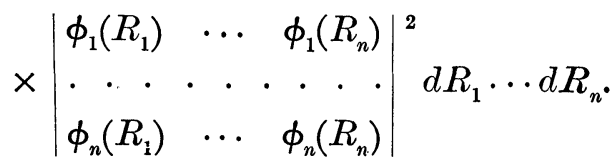

It follows that, if $k$ is nowhere negative in $R, D$ is not negative, and that if $k$ is everywhere positive in $R, D$ cannot vanish unless the functions $\phi_{1}, \phi_{2}, \cdots, \phi_{n}$ are linearly dependent. This result is obviously a generalization of the theorem known as Schwarz's inequality, in which the case $m=1, n=2$ is considered.

4. Let us consider further two sets of functions $\phi_{1}, \phi_{2}, \cdots$, $\phi_{n} ; \psi_{1}, \psi_{2}, \cdots, \psi_{n}$ satisfying the relations *

$$
\int_{R} \phi_{i} \psi_{j} d R=0 \quad(i=1,2, \ldots, n ; j=1,2, \ldots, n ; i \neq j) .
$$

If we write (1) for the sets of $n+1$ functions $\phi_{0}=f, \phi_{1}$, $\phi_{2}, \cdots, \phi_{n} ; \psi_{0}=g, \psi_{1}, \psi_{2}, \ldots, \psi_{n}$, we have for $i=j=0$ the result

$$
\begin{aligned}
\int_{R}[A f & \left.-\sum_{p=1}^{n} A_{p} \phi_{p} \int_{R} f \psi_{p} d R\right] \\
\times & {\left[A g-\sum_{p=1}^{n} A_{p} \psi_{p} \int_{R} g \phi_{p} d R\right] d R } \\
& =A\left[A \int_{R} f g d R-\sum_{p=1}^{n} A_{p} \int_{R} f \psi_{p} d R \int_{R} g \phi_{p} d R\right],
\end{aligned}
$$

where

$$
\begin{gathered}
a_{p}=\int_{R} \phi_{p} \psi_{p} d R, \quad A=a_{1} \cdot a_{2} \cdots a_{n}, \\
A_{p}=a_{1} \cdot a_{2} \cdots a_{p-1} \cdot a_{p+1} \cdots a_{n} .
\end{gathered}
$$

The further assumption that $\phi_{i}=\psi_{i}$ and that $a_{i}=1$ $(i=1,2, \ldots, n)$, i. e., that we are dealing with a normalized set

* E. g., solutions of the differential equations $L\left(\phi_{i}\right)+\lambda_{i} \phi_{i}=0, M\left(\psi_{j}\right)$ $+\lambda_{j}\left(\psi_{j}\right)=0$ (where $L$ and $M$ are adjoint differential expressions of the second order in any number of independent variables) in a closed region $R$, which vanish on the boundary of $R$. 
of orthogonal functions, leads, if applied to the case $f=g$ to Bessel's identity

(6) $\int_{R}\left[f-\sum_{p=1}^{n} \phi_{p} \int_{R} f \phi_{p} d R\right]^{2} d R=\int_{R} f^{2} d R-\sum_{p=1}^{n}\left[\int_{R} f \phi_{p} d R\right]^{2}$,

from which Bessel's inequality immediately follows.

5. Theorems analogous to those of the present note involving finite sums or infinite series in place of integrals may be proved in a similar manner.

GöTTINGKN,

June, 1909.

\section{ON THE TACTICAL PROBLEM OF STEINER.}

BY PROFESSOR W. H. BUSSEY.

(Read before the American Mathematical Society, February 24, 1906.)

THE study of tactical configurations known as triple systems had its origin in two problems proposed independently by J. Steiner $*$ and T. P. Kirkman. $\dagger$ The Steiner problem, which is the more general and includes the other, is as follows:

For what values of $n$ is it possible to arrange $n$ elements in sets of three, called triads, so that every set of two elements is contained in one and only one triad? If $n$ is a number for which there is such an arrangement in triads, are there other arrangements that cannot be obtained from it by a mere permutation of the elements? When such an arrangement in triads has been made, is it possible to arrange the $n$ elements in sets of four, called tetrads, so that no triad is contained in a tetrad and so that every set of three that is not a triad is contained in one and only one tetrad? When such an arrangement in tetrads has been made, is it possible to arrange the $n$ elements in sets of five, called pentads, so that no triad or tetrad is contained in a pentad, and so that every set of four that is not a tetrad and does not contain a triad is contained in one and only one pentad? In general, when an arrangement in $k-a d s$ has been made, is it possible to arrange the $n$ elements in sets of $k+1$, called $(k+1)-a d s$ so that no $l-a d(l \leqq k)$ is contained in a $(k+1)-a d$, and so that every set of $k$ elements that is not a

\footnotetext{
* Journal für die reine und angewandte Mathematik, vol. 45, p. 181.

†The Lady's and Gentleman's Diary for 1850. For other references to the literature of Kirkman's fifteen school girls problem see Ball's Mathematical Recreations and Essays, 4th edition, page 121.
} 